\title{
Polycystic Ovary Syndrome: It is time to change the approach in diagnosis and management
}

\author{
Carole Wehbe Chidiac, MD \\ The American Center for Psychiatry and Neurology \\ ACPN, Sunset Mall, Jumeirah Beach Road, Dubai, UAE
}

\begin{tabular}{l} 
Keywords \\
\hline PCOS \\
Lifestyle \\
Weight \\
Eating disorders \\
Mental health \\
Body image \\
Evidence-based recommendations \\
Guidelines
\end{tabular}

\begin{abstract}
Polycystic ovary syndrome (PCOS) is a common and very distressing condition in women of reproductive age. International evidence-based guideline for the assessment and management of polycystic ovary syndrome was published in 2018, with a governance including an international advisory board from six continents. The global guideline has provided a well-rounded and all-inclusive synopsis of all aspects of PCOS. Recommendations made are clear and should help eliminate areas of conflict. The addition of large sections addressing lifestyle and emotional wellbeing puts the prevalence of eating disorders in PCOS in the spotlight and strengthens the importance of looking at the patient as a whole rather than a collection of individual symptoms and problems to solve.

This commentary focuses on the evidence against many unnecessary and even harmful practices we still frequently encounter: the diagnostic criteria in teenagers, the lack of optimal ultrasound reporting, the unnecessary recommendation for non-evidencebased diets, and the harmful effects of untimely discussions disregarding women's priorities, communication preferences, beliefs culture and mental health. Last but not least, although screening for eating disorders has been recommended by many medical societies, especially before any triggering discussion about lifestyle changes, it is still rarely done in clinical practice.
\end{abstract}

Polycystic ovary syndrome (PCOS) is a common and very distressing condition in women of reproductive age characterized by a combination of hyperandrogenism and ovulatory dysfunction putting women at risk for acne, hirsutism, insulin resistance/weight gain and infertility (Williams, Mortada, \& Porter, 2016). PCOS often starts in adolescence when a girl, consulting for the irregular period or acne, is going to be easily overwhelmed, to say the least, by a diagnosis with such a long list of comorbidities: weight gain, diabetes, infertility, cancer, and clinical depression.

Inconsistent diagnostic criteria, variable provider knowledge, and contradictory data regarding comorbidities and management make it almost impossible not to have an un- favourable diagnostic experience. These factors encourage inaccurate diagnosis with both under and overdiagnosis, and the negative diagnostic experience exasperates affected women and limits timely opportunities for proper intervention.

For eating and weight disorders specialists, PCOS label is often problematic. Its presentation, weight gain for example, and recognized comorbidities, negative body image and binge eating are a recipe for disaster, made worse by the non-scientific and often harmful advice given by medical doctors. The good news is that we finally do have some evidence-based guidelines.

International evidence-based guideline for the assessment and management of polycystic ovary syndrome was

Copyright @ 2021 Carole Wehbe Chidiac. This is an open-access article distributed under the terms of the Creative Commons Attribution License (CC BY). The use, distribution or reproduction in other forums is permitted, provided the original author(s) and the copyright owner(s) are credited and that the original publication in this journal is cited, in accordance with accepted academic practice. No use, distribution or reproduction is permitted which does not comply with these terms.

Carole Wehbe Chidiac (₫) c.chidiac@americancenteruae.com

Received: 10 February 2021; Accepted: 15 February 2021; Published online: 19 February 2021. doi: 10.32044/ijedo.2021.02 
published in 2018, with a governance including an international advisory board from six continents and five guideline development groups with 63 members. The Australian Centre for Research Excellence in PCOS, funded by the National Health and Medical Research Council (NHMRC), partnered with the European Society of Human Reproduction and Embryology and the American Society for Reproductive Medicine. Thirty-seven organizations across 71 countries collaborated with 23 face to face international meetings. Sixty prioritized clinical questions involved 40 systematic and 20 narrative reviews, generating 166 practice points. "The global guideline has provided a well-rounded and all-inclusive synopsis of all aspects of PCOS. Recommendations made are clear and should help eliminate or reduce areas of conflict such as adolescent diagnosis, diagnostic criteria, and optimum fertility treatments. The addition of large sections addressing lifestyle and emotional well-being strengthens the importance of looking at the patient as a whole rather than a collection of individual symptoms and problems to solve (Jacob \& Balen, 2019)".

In this commentary, I focus on the evidence against many unnecessary and even harmful practices we still frequently encounter.

\section{Diagnostic Criteria}

The international guidelines endorse the Rotterdam PCOS diagnostic criteria in adults (i.e., two of clinical or biochemical hyperandrogenism, ovulatory dysfunction, or one of them with polycystic ovaries on ultrasound) while highlighting that ultrasound is not necessary when irregular menstrual cycles and hyperandrogenism are present, and within eight years of menarche. In other terms, in adolescents, both hyperandrogenism and ovulatory dysfunction are required for PCOS diagnosis and there is no indication for the ultrasound. Moreover, ultrasound criteria are tightened with clear protocols recommended for reporting follicle number per ovary and ovarian volume on ultrasound.

The guidelines acknowledge that actual ultrasound reports often don't meet the required criteria and that there is a need for training in careful and meticulous follicle counting per ovary, to improve reporting. Intravaginal ultrasound is recommended when possible while transabdominal ultrasound reporting is best focused on ovarian volume with a threshold of $\geq 10 \mathrm{ml}$, given the difficulty of reliably assessing follicle number with this approach. Special consideration has been given to the value and optimal timing of assessment and diagnosis of PCOS in teenagers, taking into account diagnostic challenges at this life stage, and psychosocial and cultural factors.

\section{Weight and eating disorders related comorbidities}

Although insulin resistance and predisposition for weight gain and diabetes are recognized comorbidities, "health professionals need to be aware that cardiovascular disease risk in women with PCOS remains unclear pending high-quality studies (Jacob \& Balen, 2019)". Therefore, screening for cardiovascular risk factors is an acceptable practice, but assuming that PCOS is always accompanied with high cardiovascular risk is not.

"All health professionals and women should be aware of the increased prevalence of negative body image, depression, anxiety, eating disorders and disordered eating associated with PCOS. If eating disorders and disordered eating are suspected, further assessment, referral and treatment, including psychological therapy, could be offered by appropriately trained health professionals (Jacob \& Balen, 2019)". Screening for eating disorders has been recommended by many medical societies, especially before any triggering discussion about lifestyle changes. Unfortunately, it is still rarely done in clinical practice.

\section{Lifestyle changes}

Lifestyle intervention (preferably multicomponent including diet, exercise, smoking, alcohol and behavioural strategies) should be recommended in all those with PCOS taking into consideration:

- SMART (Specific Measurable, Achievable, Realistic and Timely), goal setting.

- Psychological factors such as anxiety and depressive symptoms, body image concerns and disordered eating, to optimize engagement and adherence to lifestyle interventions.

- Adolescent and ethnic-specific body mass index and waist circumference.

- Healthy lifestyle may contribute to health and quality of life benefits in the absence of weight loss.

- Comprehensive health behavioural or cognitive-behavioural interventions to increase support, engagement, retention, adherence and maintenance of a healthy lifestyle and improve health outcomes. 
In women with PCOS, there is no or limited evidence that any specific diet type is better than another, or that there is any differential response to weight management intervention, compared to women without PCOS. Tailoring of dietary changes to food preferences, allowing for a flexible and individual approach to reducing energy intake and avoiding unduly restrictive and nutritionally unbalanced diets, are important, as per general population recommendations (Lim et al., 2019). When assessing weight, related stigma, negative body image and/or low self-esteem need to be considered and assessment needs to be respectful and considerate (Krug, Giles, \& Paganini, 2019). Beforehand, explanations on the purpose and how the information will be used and the opportunity for questions and preferences need to be provided including permission to be weighed. In conclusion, a proper assessment of PCOS is crucial as well as its timing, the information shared with the patient and the approach, taking into consideration the patient's priorities and general wellbeing.

"PCOS information should be comprehensive, evidence-based and inclusive of the biopsychosocial dimensions of PCOS across the life-span. Women's needs, priorities, communication preferences, beliefs and culture should be considered and addressed (Jacob \& Balen, 2019)". Therefore, discussing infertility and available treatment, should be timely and in response to the woman's concerns.

Ultrasound is not recommended for PCOS diagnosis in the first eight years after menarche, as it is impossible to differentiate between PCOS features and physiological changes during this period of life.

Screening for eating disorders and depression especially before discussing lifestyle changes, cannot be overemphasized, and when it comes to health promotion, the approach and the message are the same being for someone with PCOS or not.

The wide range of comorbidities requires a multidisciplinary team including gynaecologists, endocrinologists, dermatologists, psychologists and nutritionists trained in eating disorders management, with primary care physicians at the centre.
Finally, the uncertain benefits of a diagnosis need to be weighed against the potential harms of disease labelling. An increasing body of research in various medical conditions suggests that there are psychological, social, physiological and behavioural harms associated with labelling people with a medical diagnosis. One would even wonder if a PCOS diagnosis is helpful versus addressing the patient's concern without labelling (Copp et al., 2019).

\section{References}

Copp, T., Hersch, J., Muscat, D. M., McCaffery, K. J., Doust, J., Dokras, A., . . Jansen, J. (2019). The benefits and harms of receiving a polycystic ovary syndrome diagnosis: a qualitative study of women's experiences. Hum Reprod Open, 2019(4), hoz026. doi:10.1093/ hropen/hoz026

Jacob, S., \& Balen, A. H. (2019). How will the new global polycystic ovary syndrome guideline change our clinical practice? Clinical Medicine Insights: Reproductive Health, 13, 1179558119849605. doi:10.1177/1179558119849605

Krug, I., Giles, S., \& Paganini, C. (2019). Binge eating in patients with polycystic ovary syndrome: prevalence, causes, and management strategies. Neuropsychiatric Disease and Treatment, 15, 1273-1285. doi:10.2147/ndt. S168944

Lim, S., Smith, C. A., Costello, M. F., MacMillan, F., Moran, L., \& Ee, C. (2019). Barriers and facilitators to weight management in overweight and obese women living in Australia with PCOS: a qualitative study. BMC Endocrine Disorders, 19(1), 106. doi:10.1186/ s12902-019-0434-8

Williams, T., Mortada, R., \& Porter, S. (2016). Diagnosis and Treatment of Polycystic Ovary Syndrome. American Family Physician, 94(2), 106-113. 